\title{
A Case of Antimicrobial Non-compliance in an Immunocompromised Patient Resulting in Invasive Pneumococcal Disease
}

\author{
JUSTIN BAKER, CATERINA M. MIRAGLIA
}

\begin{abstract}
A 46-year-old male patient initially presented to the ED with purulent otitis media and was discharged with empirically prescribed oral and otic ciprofloxacin. Cultures of the otic discharge grew drug-resistant $S$. pneumoniae that was sensitive only to vancomycin, rifampin, and levofloxacin. Upon reviewing the culture and sensitivities, the physician attempted to contact the patient multiple times to change therapy to levofloxacin. The patient's phone line had been disconnected. Twelve days later, the patient presented with cardinal signs of meningitis and admittance to the Intensive Care Unit was required. The patient had not been compliant with the prescribed antimicrobial regimen. Imaging studies and microbiologic cultures revealed that the untreated otitis media had resulted in otomastoiditis, meningitis and bacteremia. Past medical history included recurrent ear infections, intrahepatic shunt placement, hepatic portal hypertension, and alcoholism. The patient had multiple chronic disease processes that suppressed immune function, predisposing him to invasive disease including end-stage liver disease (chronic Hepatitis $\mathrm{C}$ and alcoholic cirrhosis), and uncontrolled diabetes mellitus. The patient required extensive antimicrobial therapy to treat the infection and recovered without neurological sequelae.
\end{abstract}

ABBREVIATIONS: Streptococcus pneumoniae - S. pneumoniae, Emergency Department - ED, Diabetes Mellitus - DM, Complete Blood Count - CBC, Comprehensive Metabolic Panel - CMP, Hepatitis C Virus - HCV, Cerebrospinal fluid - CSF, Blood Urea Nitrogen - BUN, White Blood Cell - WBC, Red Blood Cell - RBC

INDEX TERMS: Streptococcus pneumoniae, otitis media, pneumococcal meningitis, patient noncompliance.
Clin Lab Sci 2015;28(3):151

Caterina M. Miraglia, DC, MLS(ASCP $)^{C M}$, Department of Medical Laboratory Science, University of Massachusetts, Dartmouth, $M A$

Justin Baker, MLS (ASCP), Massachusetts General Hospital, Boston, MA

Address for Correspondence: Caterina M. Miraglia, DC, MLS(ASCP) ${ }^{C M}$, Department of Medical Laboratory Science, University of Massachusetts Dartmouth, 285 Old Westport Road, Dartmouth, MA 02747, 508-999-8584, caterina.miraglia@umassd.edu

\section{PATIENT HISTORY}

The 46-year-old male patient had multiple ear infections as a child. He had poorly controlled insulindependent DM. The patient's liver was severely impaired due to alcohol-induced cirrhosis and chronic infection with HCV. A transjugular intrahepatic portosystemic shunt was installed 3 years prior to ED presentation to reduce portal hypertension. The portal hypertension resulted in numerous complications including Grade 2 esophageal varices and splenomegaly. Recent liver function tests had been relatively normal. The patient also suffered from gastritis, diverticulosis, hemorrhoids, pancreatitis, hypertension, neuropathy, glaucoma, anxiety, hyperlipidemia and depressive disorder. The patient admitted to smoking regularly and claimed to be abstinent from alcohol; he had spent time in a rehabilitation program three months prior to admission.

The patient initially presented to the ED with a chief complaint of ear pain. The pain had persisted for about one week, and 2 days prior to his visit to the ED, purulent discharge was produced from the ear. He reported experiencing fevers and even vomited once due 


\section{CLINICAL PRACTICE}

to extreme pain. Ibuprofen seemed to provide some pain relief. He reported recently having an upper respiratory infection with nasal congestion.

A physical examination was performed and his vitals were: normal temperature $\left(98.8^{\circ} \mathrm{F}\right)$, slightly elevated heart rate $(114$ beats $/ \mathrm{min})$, elevated blood pressure (142/68) and normal respiratory rate (18 breaths/min). All systems appeared to be normal except the right ear canal, which was filled with greenish-yellow purulent discharge and tender pinna. These findings were consistent with acute otitis media and otitis externa. Mastoid pain was demonstrated upon physical examination indicating the need for radiology consultation. Mastoiditis was evidenced by dense, sclerotic areas on CT scan. A culture of the discharge from the ear canal was performed, and laboratory panels were ordered. Panels ordered included: CMP, hepatic panel and $\mathrm{CBC}$ with differential. Table 1 displays the results of the panels ordered. Laboratory results demonstrated normal WBC counts and hyperglycemia with a normal anion gap. The decreased platelet count with increased MPV indicated that there was low/normal production with platelet destruction. Conditions associated with this include: HCV effecting marrow production or immune-meditated platelet destruction, end stage liver disease diminishing thrombopoetin synthesis, and overwhelming bacterial infection and inflammation causing platelet death. The primary conditions resulting in anemia in this patient's case were alcoholic liver cirrhosis, prescribed maintenance medications (Omeprazole, Rifamixin), $\mathrm{HCV}$, and slight hemorrhaging from esophageal varices. The patient was initially diagnosed with acute otitis media and otitis externa, and sent home. The physician had empirically prescribed Ciprodex eardrops and ciprofloxacin (Cipro) tablets to be taken daily for 10 days pending culture and sensitivities.

Initial culture of the right-ear canal discharge grew Gram-positive cocci in pairs that were Optochin sensitive by Taxo disk method, leading to identification of Streptococcus pneumoniae. Susceptibility testing revealed a multi-drug resistant strain of $S$. pneumoniae with resistance to penicillin, ceftriaxone, clindamycin, erythromycin, tetracycline and trimethoprim/ sulfamethoxazole. It was susceptible only to vancomycin, rifampin and levofloxacin. Upon reviewing the culture and sensitivities, the physician attempted to contact the patient multiple times to change therapy to levofloxacin. The patient's phone line had been disconnected.

Twelve days after the initial presentation to the ED, the patient presented with altered mental status, unintelligible speech, and violent tremors with purulent drainage from his right ear. The patient's brother had noticed his ill appearance and called emergency medical services. The patient had admitted that he did not take the prescribed ciprofloxacin daily due to provocation of heartburn symptoms. His vital signs were: increased temperature $\left(102^{\circ} \mathrm{F}\right)$, elevated heart rate $(177$, sinus tachycardia), elevated blood pressure (159/79) and elevated respiratory rate (42, pulmonary tachypnea). The right ear canal was now completely occluded with purulent discharge. Upon suctioning, it was revealed that the drainage was coming from the tympanic membrane. The patient was experiencing persistent mastoid pain and his neck was resistant to movement. Because the patient demonstrated signs of meningitis, he was started on broad-spectrum antimicrobials and an antiviral medication.

Based on the patient's presentation, differential diagnosis included: meningitis as a complication of otitis media, hepatic encephalopathy due to overwhelming infection, alcohol withdrawals, and hyperglycemic hyperosmolar non-ketotic syndrome due to uncontrolled DM and infection. Laboratory tests ordered to assess status included: CBC, CMP, blood cultures, urine cultures, lactate, and blood-alcohol level (Table 1). The electrolytes were all decreased as a result of severe osmotic diuresis.

Prothrombin Time and International Normalized Ratio (Table 1) were increased due to liver disease and the overwhelming $S$. pneumoniae infection that produces damage to blood vessels causing release of tissue factor. Increased tissue factor results in activation of the external pathway of the coagulation cascade. RBC and platelet indices were similar to the initial presentation. The peripheral blood smear demonstrated toxic granulation and vacuolated neutrophils due to severe inflammation and infection, polychromasia indicating increased RBC production, giant platelets indicating increased production as a result of destruction, and burr cells caused by increased ammonia. 


\section{CLINICAL PRACTICE}

Table 1. Laboratory Results for Initial Ear Infection and 12 days after Initial Presentation

\begin{tabular}{|c|c|c|c|}
\hline Lab Test & Reference Range & $\begin{array}{l}\text { Patient's } \\
\text { Initial } \\
\text { Presentation } \\
\text { Results }\end{array}$ & $\begin{array}{l}\text { Patient's } \\
\text { Results } 12 \\
\text { days later }\end{array}$ \\
\hline \multicolumn{4}{|l|}{ Chemistry } \\
\hline Pl Sodium & $135-145 \mathrm{mmol} / \mathrm{L}$ & $129 \mathrm{~L}$ & $131 \mathrm{~L}$ \\
\hline Pl Potassium & $3.4-4.8 \mathrm{mmol} / \mathrm{L}$ & 3.8 & $3.0 \mathrm{~L}$ \\
\hline Pl Chloride & $100-108 \mathrm{mmol} / \mathrm{L}$ & $93 \mathrm{~L}$ & $99 \mathrm{~L}$ \\
\hline $\mathrm{Pl} \mathrm{CO} 2$ & $23.0-31.9 \mathrm{mmol} / \mathrm{L}$ & 26.1 & $18.5 \mathrm{~L}$ \\
\hline Calcium & $8.5-10.5 \mathrm{mg} / \mathrm{dL}$ & - & $8.4 \mathrm{~L}$ \\
\hline Phosphorous & $2.6-4.5 \mathrm{mg} / \mathrm{dL}$ & - & $1.0 \mathrm{~L}$ \\
\hline Pl BUN & $8-25 \mathrm{mg} / \mathrm{dL}$ & $4 \mathrm{~L}$ & $5 \mathrm{~L}$ \\
\hline Pl Creatinine & $0.60-1.50 \mathrm{mg} / \mathrm{dL}$ & $.57 \mathrm{~L}$ & $.60 \mathrm{~L}$ \\
\hline Pl Glucose & $70-110 \mathrm{mg} / \mathrm{dL}$ & $356 \mathrm{H}$ & $313 \mathrm{H}$ \\
\hline eGFR & $>60 \mathrm{~mL} / \mathrm{min} / 1.73 / \mathrm{n}$ & $>60$ & $>60$ \\
\hline Albumin & $3.3-5.0 \mathrm{~g} / \mathrm{dL}$ & 3.5 & 4.0 \\
\hline Globulin & $2.3-4.1 \mathrm{~g} / \mathrm{dL}$ & $4.5 \mathrm{H}$ & $4.5 \mathrm{H}$ \\
\hline ALP & $45-115 \mathrm{U} / \mathrm{L}$ & $157 \mathrm{H}$ & $156 \mathrm{H}$ \\
\hline AST & $10-40 \mathrm{U} / \mathrm{L}$ & $93 \mathrm{H}$ & $132 \mathrm{H}$ \\
\hline ALT & $10-55 \mathrm{U} / \mathrm{L}$ & $65 \mathrm{H}$ & $75 \mathrm{H}$ \\
\hline PT & $11.0-14.0 \mathrm{sec}$ & - & $14.7 \mathrm{H}$ \\
\hline aPTT & $22.0-35.0 \mathrm{sec}$ & - & 30.0 \\
\hline Total Bilirubin & $0.0-1.0 \mathrm{mg} / \mathrm{dL}$ & .6 & - \\
\hline Pl Ammonia & $12-48 \mathrm{mcmol} / \mathrm{L}$ & - & $53 \mathrm{H}$ \\
\hline Pl Lactic Acid & $.5-2.2 \mathrm{mmol} / \mathrm{L}$ & - & $5.1 \mathrm{H}$ \\
\hline Pl Anion Gap & $3-15 \mathrm{mmol} / \mathrm{L}$ & - & 14 \\
\hline $\mathrm{BHB}$ & $<0.4 \mathrm{mmol} / \mathrm{L}$ & - & $1.9 \mathrm{H}$ \\
\hline \multicolumn{4}{|c|}{ Arterial Blood Gases } \\
\hline $\mathrm{pH}$ & $7.3-7.4$ & - & $7.47 \mathrm{H}$ \\
\hline $\mathrm{PCO} 2$ & $38-50 \mathrm{~mm} / \mathrm{Hg}$ & - & $28 \mathrm{~L}$ \\
\hline $\mathrm{PO} 2$ & $35-50 \mathrm{~mm} / \mathrm{Hg}$ & - & $63 \mathrm{H}$ \\
\hline \multicolumn{4}{|l|}{ Urinalysis } \\
\hline Urine glucose & Negative & - & $3+$ \\
\hline Urine ketones & Negative & - & $2+$ \\
\hline \multicolumn{4}{|l|}{ CBC } \\
\hline WBC & $4.5-11.0 \mathrm{th} / \mathrm{cmm}$ & 5.5 & 6.8 \\
\hline $\mathrm{RBC}$ & $4.5-5.9 \mathrm{mil} / \mathrm{cmm}$ & 4.77 & 4.73 \\
\hline HGB & $13.5-175 \mathrm{~g} / \mathrm{dL}$ & $12.4 \mathrm{~L}$ & $12.0 \mathrm{~L}$ \\
\hline HCT & $41-53 \%$ & $36.5 \mathrm{~L}$ & $35.7 \mathrm{~L}$ \\
\hline PLT & $150-400 \mathrm{th} / \mathrm{cmm}$ & $129 \mathrm{~L}$ & $114 \mathrm{~L}$ \\
\hline MCV & $80-100 \mathrm{fL}$ & $77 \mathrm{~L}$ & $76 \mathrm{~L}$ \\
\hline $\mathrm{MCH}$ & $26-34 \mathrm{pg} / \mathrm{RBC}$ & 26 & 25.4 \\
\hline $\mathrm{MCHC}$ & $31-37 \mathrm{~g} / \mathrm{dL}$ & 34 & 33.6 \\
\hline RDW & $11.5-14.5 \%$ & $16.7 \mathrm{H}$ & $16.5 \mathrm{H}$ \\
\hline MPV & $6.4-11.0 \mathrm{fL}$ & $11.1 \mathrm{H}$ & - \\
\hline Neuts & $40-70 \%$ & $69.4 \%$ & $90.0 \% \mathrm{H}$ \\
\hline Lymphs & $22-44 \%$ & $18.4 \% \mathrm{~L}$ & $2.8 \% \mathrm{~L}$ \\
\hline Abs Neuts & $1.8-7.7 \mathrm{th} / \mathrm{cmm}$ & 3.82 & 6.14 \\
\hline Abs Lymphs & $1.0-4.8 \mathrm{th} / \mathrm{cmm}$ & 1.01 & $0.19 \mathrm{~L}$ \\
\hline \multicolumn{4}{|c|}{$\begin{array}{l}\text { Plasma (Pl), Complete Blood Count (CBC), Low (L), High (H), Alkaline } \\
\text { Phosphatase (ALP), Aspartate Aminotransferase (AST), Alanine } \\
\text { Aminotransferase (ALT), White blood cells (WBC), Red Blood Cells (RBC), } \\
\text { Hemoglobin (HGB), Hematocrit (HCT), Platelets (PLT), Mean } \\
\text { Corpuscular Volume (MCV), Mean Cell Hemoglobin (MCH), Mean Cell } \\
\text { Hemoglobin Concentration (MCHC), Red cell Distribution Width (RDW), } \\
\text { Mean platelet Volume (MPV), Beta Hydroxybutyrate (BHB), Absolute } \\
\text { (Abs), Patient (Pt.) }\end{array}$} \\
\hline
\end{tabular}

A lumbar puncture was performed without complication from which cloudy CSF was obtained. CSF specimens were sent to Microbiology, Chemistry and Hematology departments. A STAT Gram stain performed on the CSF revealed Gram-positive cocci in pairs. Analysis of the CSF (Table 2), revealed an overabundance of neutrophils, RBCs protein, and glucose. This abundance of material in the CSF resulted in a slightly turbid appearance of the fluid that is normally clear and colorless. These results were consistent with bacterial meningitis and hyperglycemia. CSF glucose is normally two-thirds the plasma glucose levels. In bacterial meningitis, CSF glucose is usually decreased due to bacterial metabolism. In this case, it was high as a result of the patient's uncontrolled DM, but may have been even higher if bacteria were not present in the central nervous system. Abundant neutrophils in the CSF correlated with a meningeal bacterial infection. Increased CSF protein was as a result of the inflammatory processes of bacterial invasion and altered blood-brain barrier permeability.

The patient was at risk for life-threatening airway and circulatory compromise of multifactorial origin, as well as life-threatening central nervous system deterioration. This was cause for admittance to the Intensive Care Unit. The patient was experiencing severe dyspnea which required intubation. Dyspnea likely resulted from overwhelming infection, diabetic ketoacidosis, anemia, and meningitis.

The patient was empirically treated with vancomycin, ceftriaxone and acyclovir pending laboratory tests. Upon receiving the laboratory results and sensitivities, the meningitis was treated with a combination of vancomycin, moxifloxacin and rifampin for about two weeks while the patient was hospitalized. Since the bacteria had such high MICs to penicillin and ceftriaxone, the synergistic action of all three drugs chosen was standard therapy for treatment resistant pneumococcal meningitis. The patient was transitioned to oral levofloxacin pending discharge toward the end of his hospital stay. He was then discharged and advised to continue levofloxacin regimen for a total of six weeks to completely eliminate the infection. The patient was advised to have weekly check of BUN, creatinine, liver function tests and $\mathrm{CBC}$ to monitor patient status while on long-term antimicrobials. A follow-up appointment was scheduled prior to patient's discharge for evaluation 


\section{CLINICAL PRACTICE}

\begin{tabular}{|c|c|c|c|c|c|c|}
\hline CSF & Patient & Ref. Range & CSF Gram Stain & CSF Chemistries & Patient & Ref. Range \\
\hline $\begin{array}{c}\text { Count/Differential } \\
\text { CSF Color }\end{array}$ & White & Colorless & $\begin{array}{l}\text { Abundant } \\
\text { Neutrophils }\end{array}$ & CSF Glucose & $131 \mathrm{H}$ & $50-75 \mathrm{mg} / \mathrm{dL}$ \\
\hline CSF Turbidity & Slight & Clear & $\begin{array}{l}\text { Abundant Gram } \\
\text { Pos. Cocci In } \\
\text { Pairs }\end{array}$ & CSF Total Protein & $456 \mathrm{H}$ & $5-55 \mathrm{mg} / \mathrm{dL}$ \\
\hline Xanthochromia & None & & & & & \\
\hline $\begin{array}{l}\text { CSF Total Nucleated } \\
\text { CSF Neutrophils }\end{array}$ & $\begin{array}{c}5725 \mathrm{H} \\
98 \%\end{array}$ & $0-5 /$ cumm & & & & \\
\hline
\end{tabular}

Cerebrospinal Fluid (CSF), High (H), Reference (Ref.), Positive (Pos.)

after completing antimicrobial therapy.

\section{DISCUSSION}

The patient's immunocompromised status and noncompliance to treatment lead to development of serious infection. The patient was predisposed to acquiring the initial ear infection as a result of a recent viral upper respiratory infection, which resulted in eustachian tube blockage. Complications of acute otitis media and otitis externa include meningitis, complicated mastoiditis, and abscesses in the auditory canal, deep space infections and malignant otitis externa. The patient was at risk of having a poor prognosis as a result of his uncontrolled DM, cirrhosis, and HCV infection.

Diabetics are at an increased risk of developing bacterial infections due to their immunocompromised state. Bacterial infections that have progressed to meningitis have the ability to trigger hyperglycemia, especially in diabetics. In a four-year study of 682 patients presenting with bacterial meningitis, 69\% were hyperglycemic and 25\% severely hyperglycemic with $12 \%$ of the patients being diabetic. ${ }^{1} S$. pneumoniae accounted for $67 \%$ of the cases of meningitis in diabetics and $33 \%$ of diabetics had an infection focus of otitis/sinusitis. ${ }^{1}$ The infection acts as an inciting stressor stimulating release of hormones such as cortisol, glucagon and catecholamines that act to increase blood glucose while down-regulating insulin.

Hyperglycemic states promote immune dysfunction. During hyperglycemia, neutrophil antimicrobial activities are hindered due to factors such as inhibition of glucose-6-phosphate dehydrogenase (G6PD), leading to a decrease in NADPH. This causes suppression of antioxidant systems and inhibits oxidative burst, increases neutrophil apoptosis, decreases superoxide, decreases diapedesis and chemotaxis and decreases phagocytosis. $^{2}$ Mononuclear cells secrete less IL-1 and IL-6 in response to antigenic stimulation, which is an intrinsic cell defect or may result from tolerance. ${ }^{2}$ Uncontrolled DM is correlated with impaired CD4+ proliferation due to reduced cytokine release and/or decreased expression of cellular adhesion molecules. ${ }^{3}$ Antibody function is altered and MHC-1 expression is decreased likely as a result of glycosylation., ${ }^{2,3}$

Diabetic patients with chronic HCV infection have a more severe liver disease and increased fibrosis compared to non-diabetic patients. ${ }^{4}$ In patients with chronic HCV, DM is complicated by impaired beta cell responsiveness and/or liver damage leading to abnormal glucose metabolism/production and insulin resistance. ${ }^{4}$

Cirrhosis is another major variable that predisposes patients to infections and results in a multitude of dysfunction. The liver contains $90 \%$ of reticuloendothelial cells responsible for clearing bacteria from circulation. ${ }^{5}$ In cirrhosis, there are decreased amounts of reticuloendothelial cells and portosystemicshunting directs blood away from the liver, so antigens are not destroyed. ${ }^{5}$ Monocyte dysfunction, chemotaxis, bacterial phagocytosis and bacterial killing are drastically reduced, which correlates with the severity of liver disease. ${ }^{5}$ C3 and C4 deficiency exacerbate these issues, resulting in decreased opsonization and complement-mediated cell death. The role of complement is extremely important and deficiencies promote the transition of pneumococcal colonization to invasive disease. $^{6}$

Streptococcus pneumoniae is the major etiologic agent implicated in multiple infectious disease processes including upper respiratory infections, meningitis, bacteremia and pneumonia. The incidence of invasive pneumococcal disease is high in populations with 


\section{CLINICAL PRACTICE}

compromised immune systems. S. pneumoniae can be part of the upper respiratory tract flora in human carriers, and may be transmitted person to person via respiratory droplets or by autoinoculation. Carriage rates are high among children and those in crowded areas such as prisons, long-term care facilities, and hospitals. ${ }^{7}$ Individuals with immunocompromised states such as DM, chronic liver disease, alcoholics, young children, and elderly are most at risk for infection. These Gram-positive cocci, also known as pneumococci, have a variety of virulence factors and mechanisms to evade host defenses. The pathogenic species are encapsulated which provides resistance to host phagocytosis, prevents activation of the alternative complement pathway, and provides protection from mucus while adhering to epithelial cells. There are over 90 capsular serotypes, leading to poor host immunity. Pneumolysin and autolysin are potent intracellular toxins released upon lysis of the pneumococcus that potentiate inflammation, suppress host defenses, and result in cellular death. Cell wall components are potent initiators of complement and inflammatory mediators, facilitating systemic invasion and increasing the risk of neurologic sequelae. Hyaluronidase facilitates penetration and invasion via degradation of host connective tissue. Phosphorylcholine in the cell wall binds to receptors of the cells in the meninges and blood vessel walls stimulating the target cell to phagocytize the bacteria, enabling evasion of detection and passage across the blood-brain barrier. ${ }^{8}$

At the time of local colonization, the pathogenic $S$. pneumoniae disperses from the epithelium to the bloodstream where it releases exotoxins along with other pathogenic stimuli to initiate inflammation, complement, and the coagulation cascade. The release of host inflammatory mediators such as IL-1, IL-6, and TNF, leads to increased vascular permeability, emigration of leukocytes and elevated cellular metabolism, which aids in transgression of the bloodbrain barrier. The pneumococci take up residence in the central nervous system, multiplying rapidly due to minimal local host-defense mechanisms. The bacteria are presented to local circulating antigen-presenting cells and resident microglial cells with subsequent inflammation of the meninges and neutrophil recruitment. $^{6}$ Another mechanism of meningeal invasion involves direct extension from the inner ear and mastoid air cells into the sub-arachnoid space. ${ }^{7}$
Ciprofloxacin should not be used to treat pneumococcal infections since it has limited Gram-positive and antipneumococcal activity. The newer generation fluoroquinolones such as levofloxacin and moxifloxacin have much greater activity against pneumococci. The CDC considers drug-resistant $S$. pneumoniae a "serious hazard" and they estimate $30 \%$ of severe pneumococcal cases are resistant to one or more clinically relevant antibiotics, complicating treatment for almost 1.2 million cases yearly. ${ }^{9}$

Patient non-compliance to treatment of acute infections is a common occurrence. It has been estimated that as many as $40 \%$ of outpatients do not adhere to the prescribed antimicrobial regimen. Other sources estimate that this number may be even greater, possibly as high as $70 \% .^{10}$

Common causes of non-compliance include patient beliefs; for example, belief that an antimicrobial is unnecessary, or concern over interactions with other drugs and/or alcohol. Other causes include cost, frequency of dosage, treatment duration, side effects, patient forgetfulness, and rapid improvement of symptoms. Rapid improvement of symptoms is the most common reason for non-compliance. When patients begin to feel better, they believe they do not need to finish the medication as prescribed. ${ }^{10}$

There are major consequences to non-compliance to an antimicrobial regimen. These include treatment failure, recurrent infections and complications, need for additional office visits, testing and treatment, addition of substantial cost to the healthcare system, possible death of the patient, and development of antimicrobialresistant microorganisms. ${ }^{10}$

Studies show that compliance is not dependent upon age, gender, marital status, education, income, or social class. Complexity of the dosage schedule has been shown in studies to directly effect compliance. The more complex the treatment regimen, the less likely the patient will adhere to it. It is therefore important for the physician to, if possible, prescribe a treatment regimen that is less complex and just as effective as a more complex regimen. If this is not possible, the physician should talk with the patient to find events during the patient's day that will serve as reminders for them to take their medication. Patient education is also an 


\section{CLINICAL PRACTICE}

important method in improving compliance. Patients need to be educated on the risks of misuse of antimicrobials, the benefits of compliance, and not to expect an antimicrobial for every infection, as many are not bacterial in origin. ${ }^{10,11}$

\section{CASE CONCLUSION}

This patient's case demonstrated the ability of $S$. pneumoniae to cause invasive disease in patients with compromised immune systems and in patients who do not adhere to a prescribed treatment regimen. Various virulence factors allow the organism to evade host immune defenses, potentiate inflammatory disease and transgress a host's natural protective barriers. Accurate assessment of the patient led to the appropriate acute care actions. The risk of neurologic sequelae is very high with pneumococcal meningitis and in immunocompromised patients, but fortunately the patient did not experience this complication and ultimately recovered. Resistant $S$. pneumoniae infections are uncommon occurrences that require tailored antimicrobial therapy in order to rid the patient of infection. Patient non-compliance to antimicrobial therapy is a serious problem, especially at a time when there is an increased emergence of antimicrobial resistant microorganisms. Patient education on the risks of misuse of antimicrobial therapy both to themselves as well as to the community is a key method in solving the problem.

\section{ACKNOWLEDGEMENTS}

A special thanks to Laura Listro at Massachusetts General Hospital.

\section{REFERENCES}

1. Schut ES, Westendorp WF, de Gans J, Kruyt ND, Spanjaard L, Reitsma JB, van de Beek D. Hyperglycemia in bacterial meningitis: a prospective cohort study. BMC Infect Dis. 2009 May 8;9:57. doi:10.1186/1471-2334-9-57.

2. Casqueiro J, Casqueiro J, Alves C. Infections in patients with diabetes mellitus: a review of pathogenesis. Indian J Endocrinol Metab. Mar 2012;16:S27-36. doi: 10.4103/2230-8210.94253.

3. Geerlings $S$, Hoepelman A. Immune dysfunction in patients with diabetes mellitus. FEMS Immunol. Med. Microbiol. 1999 Jul 25;26:259-65. doi: 10.1111/j.1574-695X.1999.tb0139.

4. Elhawary EI, Mahmoud GF, El-Daly MA, Mekky FA, Esmat GG, Abdel-Hamid M. Association of HCV with diabetes mellitus: an Egyptian case-control study. Virol J. 2011 July 26; 8:367. doi: 10.1186/1743-422X-8-367.

5. Bonnel AR, Bunchorntavakul C, Reddy KR. Immune dysfunction and infections in patients with cirrhosis. Clin Gastroenterol Hepatol. 2011 September 9;9:727-38. doi: 10.1016/j.cgh.2011.02.031.

6. Mook-Kanamori BB, Geldhoff M, van der Poll T, van de Beek D. Pathogenesis and pathophysiology of pneumococcal meningitis. Clin Microbiol Rev. 2011 Jul; 24(3):557-91. doi: 10.1128/CMR.00008-11.

7. Kumar V, Abbas A, Aster J. Robbins basic pathology. $9^{\text {th }}$ ed. Philadelphia: Saunders/Elsevier; 2013.

8. Bauman RW. Microbiology with diseases by body system. $3^{\text {rd }}$ ed. San Francisco: Pearson; 2012.

9. CDC.gov. [Internet]. Atlanta: Centers for Disease Control and Prevention. Antibiotic resistance threats in the United States 2013. [cited 2014 Jul 1]. Available from: http://www.cdc.gov/ drugresistance/threat-report-2013/ pdf/ar-threats-2013-508.pdf

10. Kardas P. Noncompliance in current antibiotic practice. Infect Dis Clin Pract. 2006 Jul; 14(4):S11-14. doi: 10.1097/01.idc.0000230544.11499.8b

11. J Cockburn, RW Gibberd, AL Reid, RW Sanson-Fisher. Determinants of non-compliance with short term antibiotic regimens. $\mathrm{Br}$ Med J (Clin Res Ed). 1987 Oct 3; 295(6602):814-8. 